\author{
Nicolas Grenier \\ Francesco Sardanelli \\ Christoph D. Becker \\ Jerzy Walecki \\ Guy Sebag \\ David John Lomas \\ Gabriel P. Krestin
}

\section{Development of molecular imaging in the European radiological community}

Received: 3 July 2008

Revised: 4 November 2008

Accepted: 25 November 2008

Published online: 20 January 2009

(C) European Society of Radiology 2009

\author{
N. Grenier $(\square)$ \\ Groupe Hospitalier Pellegrin, \\ Imagerie diagnostique et thérapeutique \\ de l'adulte, \\ Place Amélie Raba-Léon, \\ Bordeaux, 33076, France \\ e-mail: nicolas.grenier@chu-bordeaux. \\ fr \\ F. Sardanelli \\ IRCCS Policlinico San Donato- \\ Radiology, University of Milan, \\ Milan, Italy \\ C. D. Becker \\ Diagnostic and Interventional \\ Radiology, Geneva University \\ Hospital, \\ Geneva, Switzerland
}

\author{
J. Walecki \\ Department of Radiology, \\ Medical University of Bialystok, \\ Bialystok, Poland \\ G. Sebag \\ Service d'imagerie médicale, \\ Hôpital Robert Debré, \\ Paris, France \\ D. J. Lomas \\ Department of Radiology, \\ Addenbrooke's Hospital, \\ Cambridge, UK \\ G. P. Krestin \\ Department of Radiology, Erasmus \\ MC, University Medical Center \\ Rotterdam, \\ Room D219, \\ Rotterdam, Netherlands
}

\begin{abstract}
The recent and concomitant advances in molecular biology and imaging for diagnosis and therapy will place in vivo imaging techniques at the centre of their clinical transfer. Before that, a wide range of multidisciplinary preclinical research is
\end{abstract}

already taking place. The involvement of radiologists in this new field of imaging sciences is therefore absolutely mandatory during these two phases of development. Achievement of such objectives requires the refinement of strategy within the European radiological community and the European Society of Radiology (ESR) will have to drive a number of actions to stimulate the younger generation of radiologists and to facilitate their access to knowledge. For that purpose, a molecular imaging (MI) subcommittee of the ESR Research Committee based on a group of involved radiologists will be constituted to develop contacts with other constitutive committees and associated societies to provide proposals to our community.

Keywords Molecular imaging · Radiology policy · Cellular imaging · Drug delivery

\section{Introduction}

In 2007 the newly formed ESR Executive Council asked the Research Committee of ESR to set up a working group with the objective to develop a strategic paper on the development and implementation of molecular imaging research into the European radiological community. The members of the working group nominated by the ESR Research Committee were: N. Grenier (France, chair of the working group), F. Sardanelli (Italy), C. Becker (Switzerland), J. Walecki (Poland), G. Sebag (France), D. Lomas (UK) and G.P. Krestin (Netherlands, Chair of the ESR Research Committee). The strategy paper adopted by the ESR Research Committee was subsequently widely discussed within ESR and its Educational, Subspecialty and National Societies Committees. ESR has embraced and started to adopt many of the recommendations of this strategy paper as described in the present article. 


\section{General considerations}

Molecular imaging (MI) can be defined as:

- The science for in vivo extraction of biological information resolved in time and space [1]

- The techniques to, directly or indirectly, monitor and record the spatiotemporal distribution of molecular and cellular processes for biochemical, biological, diagnostic or therapeutic applications [2]

- The visual representation, characterization and quantification of biological processes at the cellular and subcellular levels within intact living organisms [3]

The recent arrival of molecular imaging research in the radiological community is the result of concomitant development in cellular and molecular biology, in genomics and proteomics, in nanotechnologies and increased sophistication of all our imaging technologies, combined with a large diversification in new contrast agents and probes. Until now, a radiological examination was performed when a disease was suspected to have determined a change in anatomy, structure or function in one or several organs or systems. This event occurs relatively late in the disease process. MI could allow a substantial diagnostic anticipation with a tremendous impact on clinical practice and screening programmes.

Combining imaging techniques with biological targets is not a new concept because it has already been performed for many years in the world of nuclear medicine (essentially with the development of positron emission tomography, PET). However, the use of such a combination within the radiological community raises difficulties essentially due to the level of basic knowledge required. Even if molecular imaging has already shown potential in a few clinical applications, it is still considered a field of basic research on small animals.

The future potential of MI is tremendous and has been emphasized in many reports [4-8]; the purpose of this article, however, was not to develop all these aspects in detail, but the reader should be aware that the opportunities of MI are numerous and should have considerable impact on:

- All phases of biomedical research

- Disease knowledge (to provide biomarkers of disease processes, to improve the detection of diseases at a presymptomatic stage, to improve the characterisation of phenotypes of diseases based on biological and biochemical information, to improve the staging of diseases etc.)

- Biological events (to quantify biological processes as inflammation, apoptosis, angiogenesis etc.; to detect signal transduction, gene expression, expression of receptors, synthesis of proteins etc.)
- Cellular events (to monitor cell migration, cell differentiation, distinction between stem cells and non-stemcell in a tumour cell population etc.)

- Drug discovery (to help in defining stratified study groups based on specific biomarkers, to perform longitudinal studies in animals, to enhance understanding of the pathways of drug action and effectiveness, to provide information on the optimal timing and dosing of drugs, to provide earlier surrogate markers of drug effectiveness etc.)

- Treatment assessment (to monitor the delivery of gene or drug by new "nanovehicles", to control spatially gene expression etc.)

- The further development of "individualized medicine"

Molecular imaging must be considered as a major opportunity and natural evolution for radiologists owing to the increasing spatial resolution of our current imaging techniques, and to the development of hybrid imaging systems and of new probes:

- It opens wide new fields of research and brings new potential clinical applications of medical imaging

- It places - as never before-radiological imaging at the centre of diagnosis and patient management

- It could reorient, in the future, "medical imaging" (and medical sciences in general) with its traditional segmentation in organ-based subspecialties, towards "biomedical imaging" with a more transversal segmentation based on biological processes

- It will change the content and structure of the future educational programmes for our young radiologists

- It will cause education and research to become more multidisciplinary and, in the longer term, it will change our clinical practice

Therefore, it now becomes essential for radiologists to play an important role in the field of MI. Their involvement will have to focus on basic and preclinical developments, and prepare the speciality to be ready, in the near future, for clinical translation. However, the introduction of this process will require a much longer development period than was required when previous new technologies were introduced. It should follow a more stepwise approach from imaging laboratories, in which MI imaging is initially developed, to other academic institutions, major hospitals, to finally reach outpatient centres.

Historically, the preclinical development of X-ray, ultrasound, CT and MR imaging was accomplished by engineers and physicists. Radiologists had those tools in their hands for historical reasons and they contributed more in the clinical refinement of the application field than in the preclinical work. For MI, it will be similar: the development of new and even hybrid imaging techniques is more within the field of physicis than radiology. However, application of these techniques to biological disease 
processes through hypothesis testing should be a major topic for our future activity.

It is a well-known phenomenon that domination of a new technique belongs to those who became most involved in its research and development phases. As in the past for other imaging techniques, MI may become a source of "turf battles" between specialties. Clinical specialties are also developing strategies to get involved in this field which is mandatory for the development of their own research topics. This competition will increase in accordance with its dissemination into clinical practice [9].

Radiologists have accumulated knowledge and expertise in most imaging technologies, in pathophysiology and evolution of diseases, in patient management and in imageguided interventions. This expertise should help the radiological community in mastering the challenge of introducing $\mathrm{MI}$ into clinical practice.

For all these reasons, ESR has decided to play a key role in the development of MI in radiology by supporting education, by increasing radiologists' involvement and by promoting this translation in — and even outside - the medical community.

\section{Impact on the organisation of radiology}

Modern medicine is progressively moving towards dealing with specific types of diseases (degenerative, inflammatory, infectious, cancer etc.) in a multidisciplinary way. Therefore, imaging research could parallel such new developments with a possible impact on segmentation of clinical imaging. Development of research and educational programmes in that field within academic departments requires one to build up "multidisciplinary imaging teams" with chemists (to develop and provide new tracers and agents for identification of specific targets or delivery of products), cellular and molecular biologists (to define the new functional imaginable targets), physicists (to optimise the existing imaging systems and to develop new techniques), computer scientists (to develop software for signal and image processing and for in vivo quantification of biological processes) and scientists from other disciplines depending on the systems being developed (optics, nanotechnology, engineering etc.).

\section{Impact on education}

A basic level of knowledge should be incorporated into radiology training programmes for all radiology residents regarding basic principles of molecular and cellular biology, of optical imaging, PET and combined (hybrid) modalities (PET-CT, SPECT-CT, PET-MR), of specific and nonspecific targeted and polyfunctional agents, and of methods of cell labelling and cell targeting.

Considering the important prerequisite necessary for embracing the topic, the ESR Educational Committee will also reconsider the content of the full curriculum to integrate some flexibility for the younger radiologists who wish to subspecialise in this field. A specific educational programme should be developed as a 1- to 2-year programme. The topics included in this programme could be also offered on a modular basis (as an MI masters programme) including workshops or seminars. Details of future education programmes still have to be defined but a close collaboration between imaging specialists (radiologists and NM specialists) and biologists is very important for that purpose.

If possible, this programme should be integrated within a specific research fellowship, for a 1- or 2-year duration and be delivered only in centres of excellence accredited by ESR. Some already exist and others, in collaboration with the European Institute for Biomedical Imaging Research (EIBIR), will have to be identified and evaluated using the database of EIBIR institutions. An important prerequisite for academic radiology departments to develop MI is to provide attractive long-term academic positions for basic researchers.

\section{Centres of excellence in MI}

An update on radiological forces in MI will be established (experts involved in the field, centres involved in MI research programmes, centres with established MI educational programmes, platforms dedicated to MI etc.). Those centres of excellence will be defined based on the database of EIBIR institutions. The multidisciplinary cooperation and a multimodal research chain from basic to translational and clinical research will be important items for ESR recognition and accreditation. Radiologists have to develop their own scientific questions and integrate them into the work of those centres. The centres will have to be integrated in networks allowing exchange of knowledge and researchers and make use of the already existing European Commission grant support within the 7th Framework Program (such as the recently awarded ENCITE programme for cell imaging).

\section{Promotion of MI and communication}

ESR should promote a specifically oriented communication programme emphasizing promotion of molecular imaging within the radiological and medical communities. Grants for scholarships and short-term fellowships for young radiologists interested in MI research should be provided. Pursual of MI programmes should be promoted in academic departments. Diffusion of knowledge should be planned with lectures given in plenary sessions of main European radiology meetings, publication of scientific articles and reviews in main European radiology journals and communication on the topic through the medical media.

Effort should also be made regarding communication with authorities and regulatory bodies to help the European authorities to build up regulations adapted to diffusion, 
validation and, at a later stage, reimbursement for these new technologies, and to coordinate efforts in MI within the global international community.

\section{Financial support}

Considering the long time required for diffusion, validation and reimbursement of these technologies, adequate funding will be necessary. The ESR MI grant funded by NCI and offered to young European researchers by the Memorial Sloan Kettering Cancer Centre in New York under the leadership of Prof. H. Hricak is an example of efficient transatlantic sponsorship and collaboration. Many other opportunities could be implemented in collaboration with ESOR and EIBIR and based on European research grants and industrial sponsorship. Moreover, ESR has recently established the EIBIR Fund, a fund-raising activity that will enable us to support research education to personal and institutional donations.

\section{Role of groups and societies}

ESR, together with subspecialty societies, will help to identify early leaders of this developing area amongst younger radiologists. They should be positioned and able to drive and influence the development of MI programmes from a position of knowledge and experience in their specific area of expertise. The ESOR has the potential to offer substantial help for promoting European courses in MI and/or annual workshops.

Amendments to the educational curriculum for radiologists as well as the content of an optimal research fellowship MI for young radiologists are necessary and should also be defined by ESR Educational and Research Committees together.

Finally, help from industry is welcome and presently provided for the establishment and initial organization of EIBIR. In future, donations to the EIBIR Fund will be used for supporting diffusion and education of MI-related topics. Considering the interest of industry in this field, common initiatives and cooperation on grant applications with societies of manufacturing engineers and industrial R\&D laboratories will intensify the translation of inventions into clinical practice.

\section{Summary}

In summary, the recent and concomitant advances in molecular biology and in imaging for diagnosis and therapy will place in vivo imaging techniques at the centre of their clinical transfer. Before that, a wide range of multidisciplinary preclinical research is already taking place. Involvement of radiologists in this new field of imaging sciences is therefore absolutely mandatory during these two phases of development. Achievement of such objectives requires the refinement of strategy within the European radiological community. Therefore, ESR will have to drive a number of actions to stimulate the younger generation of radiologists and to facilitate their access to knowledge. For that purpose, a molecular imaging subcommittee of the ESR Research Committee based on a group of involved radiologists will be constituted to develop contacts with other constitutive committees and associated societies to provide proposals to our community.

\section{References}

1. Zerhouni EA (2005) US biomedical research: basic, translational, and clinical sciences. JAMA 294:1352-1358

2. Thakur M, Lentle BC (2005) Report of a summit on molecular imaging. Radiology 236:753-755

3. Massoud TF, Gambhir SS (2003) Seeing fundamental biological processes in a new light. Genes Dev 17:545-580
4. Ehman RL, Hendee WR, Welch MJ et al (2007) Blueprint for imaging in biomedical research. Radiology 244:12-27

5. Hoffman JM, Gambhir SS (2007) Molecular imaging: the vision and opportunity for radiology in the future. Radiology 244:39-47

6. Weissleder R, Mahmood U (2001) Molecular imaging. Radiology 219:316-333

7. Wu JC, Bengel FM, Gambhir SS (2007) Cardiovascular molecular imaging. Radiology 244:337-355
8. Rudin M, Weissleder R (2003) Molecular imaging in drug discovery and development. Nat Rev 2:123-131

9. Hillman BJ, Neiman HL (2002) Translating molecular imaging research into radiologic practice: summary of the proceedings of the American College of Radiology Colloquium, April 22-24, 2001. Radiology 222:19-24 